\title{
Scrolls containing binary curves
}

\author{
Marco Franciosi
}

\begin{abstract}
We study families of scrolls containing a given rational curve and families of rational curves contained in a fixed scroll via a stratification in terms of the degree of the induced map onto $\mathbb{P}^{1}$ and we prove that there is no rational normal scroll of minimal degree and of dimension $\leq \frac{n}{2}$ containing a general binary curve in $\mathbb{P}^{n}$.
\end{abstract}

keyword: algebraic curve, rational normal scroll, binary curve

Mathematics Subject Classification (2010) 14H10, 14H51, 13D02, $14 \mathrm{~N} 25$

\section{Introduction}

A classical result in birational geometry is that a nondegenerate irreducible variety $X$ in $\mathbb{P}^{n}$ has degree at least $\operatorname{codim}(X)+1$ and moreover if equality holds then either $X$ is a rational normal scroll, or it is a cone over the Veronese surface $\mathbb{P}^{2} \hookrightarrow \mathbb{P}^{5}$, or it is a quadric hypersurface (see [7, Theorem 1]) .

The aim of this paper is the study of families of scrolls containing a fixed scheme (in particular a given rational curve) and families of rational curves contained in a given scroll by considering the incidence correspondence of such families.

We point out that by a rational normal scroll we mean not only the variety $\mathbb{F}\left(a_{1}, \ldots, a_{d}\right)$, but also its birational image in $\mathbb{P}^{n}$, which may be singular and that by a family of rational normal scrolls of dimension $d$ in $\mathbb{P}^{n}$ we mean a subscheme of $\operatorname{Hilb}^{p}\left(\mathbb{P}^{n}\right)$, where $p=p(h)$ is the Hilbert polynomial of any irreducible normal scroll of dimension $d$ and degree $n-d+1$ in $\mathbb{P}^{n}$.

Bogomolov and Kulikov in [2] considered the surface case, by studying the subschemes of $\operatorname{Hilb}\left(\mathbb{P}^{n}\right)$ whose points correspond to the surface scrolls, resp. to cones over rational normal curves spanning $\mathbb{P}^{n}$.

Here we firstly give an overview of families of rational normal scrolls satisfying some given conditions (see Thm. 3.1 which is a generalisation to every dimension of [2, Proposition 2]) and secondly we study in detail the case in which it is given a rational normal curve $\Gamma$ passing through a set $S$ of $n+2$ points in linear general position in $\mathbb{P}^{n}$. To be more precise fixing $k \in \mathbb{N}$ we consider the family $\mathcal{F}(S, k)_{\underline{a}}$ of scrolls of dimension $d=\frac{n}{2}$ containing $\Gamma$ such that the induced map $\Gamma \rightarrow \overline{\mathbb{P}}^{1}$ has degree $k$ (see Prop. 4.9). We study the main 
properties of such family and we show that for two distinct integers $h, k$ such that $k+h>d$ then $\operatorname{dim}\left(\mathcal{F}(S, k)_{\underline{a}} \cap \mathcal{F}(S, h)_{\underline{a}}\right) \leq 2 n-3$ (see Prop. 4.10).

On the other side, one of the characterisations of scrolls is that they contain many rational curves. Here we study the family of rational normal curves passing through $n+2$ fixed points and contained in a fixed scroll scroll $\mathbb{F}$ of dimension $d$. In Prop. 4.6 we show that such family has dimension at most $\#(S \cap \operatorname{Sing}(\mathbb{F}))$, whereas in Prop. 4.8 we show that if such family contains more than one single curve then it has strictly positive dimension.

As an application of the above results, in section 5 we study families of scrolls containing binary curves. A binary curve is a stable curve $C$ consisting of two irreducible curves, both isomorphic to $\mathbb{P}^{1}$, intersecting transversally in $n+2$ distinct points (hence of arithmetic genus $p_{a}(C)=n+1$ ). In the nonhyperelliptic case $\left|K_{C}\right|$ embeds $C$ as the union of two rational normal curves of degree $n$ in $\mathbb{P}^{n}$ passing through $n+2$ points in linear general position, so that we can identify $C$ with its image in $\mathbb{P}^{n}$. We want to point out firstly, as shown by Colombo and Frediani in [4, the usefulness of binary curves in studying moduli problem of curves of genus $p_{a}(C)$ and secondly that the existence of a scroll containing the curve $C$ forces the non-vanishing of higher order syzygies of the ideal $I_{C}$ (see e.g. [1]).

Theorem 5.5 shows that given a general binary curve $C=\Gamma_{1} \cup \Gamma_{2}$ in $\mathbb{P}^{n}$, then there is no rational normal scroll of minimal degree and of dimension $\leq \frac{n}{2}$ containing $C$.

\section{Acknowledgements}

The author is grateful for support by the PRIN project 2015EYPTSB_010 "Geometry of Algebraic Varieties" of italian MIUR.

The author would like to thank Elisa Tenni for deep and stimulating discussions on these arguments.

\section{Notation and conventions}

We work over the field $\mathbb{C}$. We will consider subschemes of $\mathbb{P}^{n}$, assuming $n \geq 3$.

Throughout this paper by $S$ we will denote a set consisting of $n+2$ points in linear general position in $\mathbb{P}^{n}$, by $\Gamma$ we will denote a rational normal curve in $\mathbb{P}^{n}$, and by $\mathbb{F}$ a rational normal scroll in $\mathbb{P}^{n}$.

To clarify our point of view we recall the description of rational normal scroll as shown in [13, Chapter 2].

Let $\underline{a}$ be a multi-index of $d$ non-negative integers $\underline{a}=\left\{a_{1}, \ldots, a_{d}\right\}$ such that $\sum a_{i}=n-d+1$. By $\mathbb{F}_{\underline{a}}=\mathbb{F}\left(a_{1}, \ldots, a_{d}\right)$ we mean the scroll $\mathbb{P}\left(\bigoplus_{i=1}^{d} \mathcal{O}_{\mathbb{P}^{1}}\left(a_{i}\right)\right)$. It has coordinates $\left(y_{1}, \ldots, y_{d}\right),\left(t_{0}, t_{1}\right)$ and the map $\mathbb{F}\left(a_{1}, \ldots, a_{d}\right) \rightarrow \mathbb{P}^{1}$ corresponds to the coordinates $\left(t_{0}, t_{1}\right)$. Moreover the divisor class group is a free group generated by $L$, the pullback of $\mathcal{O}_{\mathbb{P}^{1}}(1)$, and $M$ a relative hyperplane, which is 
characterised by the property that the locus $\left(y_{i}=0\right)$ is a divisor in the class $-a_{i} L+M$ (equivalently $M$ is the class of any monomial $t_{0}^{b} t_{1}^{c} y_{i}$ with $b+c=a_{i}$ ). If $a_{i}>0$ for some $i$, then $|M|$ is a base point free system which induces a map $\varphi_{|M|}: \mathbb{F}\left(a_{1}, \ldots, a_{d}\right) \rightarrow \mathbb{P}^{n}$, birational onto its image $\mathbb{F}$; if moreover $a_{i}>0$ for every $i, \varphi_{|M|}$ is an embedding. Since no confusion should arise, we will call rational normal scroll not only the variety $\mathbb{F}\left(a_{1}, \ldots, a_{d}\right)$, but also its birational image $\mathbb{F}$ in $\mathbb{P}^{n}$. On the contrary if $\mathbb{F} \subset \mathbb{P}^{n}$ is a rational normal scroll obtaining by joining points on $d$ distinct rational normal curves lying in complementary linear subspaces (see e.g. [10, Example 8.26]), then there exists a unique smooth rational normal scroll $\mathbb{F}\left(a_{1}, \ldots, a_{d}\right)$ with $a_{1} \geq 0$ for every $i$, such that $\mathbb{F}$ is the birational image of $\mathbb{F}\left(a_{1}, \ldots, a_{d}\right), M=\mathcal{O}_{\mathbb{P} n}(1)_{\mathbb{F}}$ and $L$ the pullback of $\mathcal{O}_{\mathbb{P}^{1}}(1)$ via the standard projection $\pi: \mathbb{F} \rightarrow \mathbb{P}^{1}$.

Let $\mathbb{F} \subset \mathbb{P}^{n}$ be a rational normal scroll of dimension $d$ and degree $n-d+1$. Let $p$ be its Hilbert polynomial. By a family of rational normal scrolls of dimension $d$ and degree $n-d+1$ in $\mathbb{P}^{n}$ we mean a subscheme of $\operatorname{Hilb}^{p}\left(\mathbb{P}^{n}\right)$.

Throughout the paper e will constantly use the following notation for families of scroll:

$\mathcal{G}$ the family of rational normal curves in $\mathbb{P}^{n} ;$

$\mathcal{G}(Y)$ the family of rational normal curves in $\mathcal{G}$ containing a given scheme $Y \subset$ $\mathbb{P}^{n}$

$\mathcal{G}^{\vee}(\mathbb{F})$ the family of rational normal curves in $\mathcal{G}$ contained in a given scroll $\mathbb{F} \subset \mathbb{P}^{n}$;

$\mathcal{G}^{\vee}(\mathbb{F}, k)$ the family of rational normal curves $\Gamma$ in $\mathbb{F}$ such that the induced map $\Gamma \rightarrow \mathbb{P}^{1}$ has degree $k$;

$\mathcal{F}$ the family of rational normal scrolls of dimension $d$ and degree $n-d+1$ in $\mathbb{P}^{n}$;

$\mathcal{F}(Y)$ the family of rational normal scrolls in $\mathcal{F}$ containing a given scheme $Y \subset$ $\mathbb{P}^{n}$

$\mathcal{F}_{\underline{a}}$ the family of rational normal scrolls in $\mathbb{F}$ such that $\mathbb{F} \cong \varphi_{|M|}\left(\mathbb{F}\left(a_{1}, \ldots, a_{d}\right)\right)$;

$\mathcal{F}_{0}$ the family of balanced scrolls, i.e. the family $\mathcal{F}_{\underline{a}}$ corresponding to the multi-index $\underline{a}$ such that $\left|a_{i}-a_{1}\right| \leq 1$; note that this $\underline{a}$ is unique up to permutation;

$\mathcal{F}(Y)_{\underline{a}}$ the family $\mathcal{F}(Y) \cap \mathcal{F}_{\underline{a}} ;$

$\operatorname{Aut}(\underline{a})$ the group $\operatorname{Aut}\left(\bigoplus_{i=1}^{d} \mathcal{O}_{\mathbb{P}^{1}}\left(a_{i}\right)\right)$. 


\section{Families of rational normal scrolls}

In this section we point out a theorem which collects some well known results on families of scrolls and of rational normal curves in $\mathbb{P}^{n}$. We believe that most of these results were known since the 19 th century, but we were not able to find an adequate reference.

Theorem $3.1 \quad$ (a) The dimension of the family $\mathcal{F}$ of rational normal scrolls of dimension $d$ and degree $n-d+1$ in $\mathbb{P}^{n}$ is $n^{2}+2 n-2-d^{2}$.

(b) The dimension of the family $\mathcal{F}_{\text {a }}$ of rational normal scrolls in $\mathcal{F}$ birational to the scroll $\mathbb{F}(\underline{a})=\mathbb{F}\left(a_{1}, \ldots, a_{d}\right)$ is $n^{2}+2 n-2-d^{2}-\left(\operatorname{dim} \operatorname{Aut}(\underline{a})-d^{2}\right)$.

(c) The family $\mathcal{F}_{0}$ corresponding to scrolls $\mathcal{F}_{\underline{a}}$ such that $\left|a_{i}-a_{1}\right| \leq 1 \forall i>1$ is dense in the family $\mathcal{F}$ of rational normal scrolls of dimension $d$ and degree $n-d+1$ in $\mathbb{P}^{n}$.

(d) The dimension of the family $\mathcal{G}$ of rational normal curves in $\mathbb{P}^{n}$ is $n^{2}+$ $2 n-3$.

(e) Let $S$ be a set of $n+2$ points in linear general position in $\mathbb{P}^{n}$. Then the family $\mathcal{G}(S)$ of rational normal curves containing $S$ has dimension $(n-1)$.

(f) Let $S$ be a set of $n+2$ points in linear general position in $\mathbb{P}^{n}$. Let $\mathcal{F}(S)_{\underline{a}}$ be the family of rational normal scrolls in $\mathcal{F}_{\underline{a}}$ containing $S$. Then

$$
\operatorname{dim} \mathcal{F}(S)_{\underline{a}}=(n+2) \cdot d-\left(d^{2}+2\right)-\left(\operatorname{dim} \operatorname{Aut}(\underline{a})-d^{2}\right)
$$

Proof. Fix a suitable multi-index $\underline{a}$ and assume at first that $d \neq 2$ or $d=2$ and $a_{1} \neq a_{2}$. The points of $\mathcal{F}_{a}$ parametrise the images of all possible morphisms $g: \mathbb{F}(\underline{a}) \rightarrow \mathbb{P}^{n}$ given by the complete linear series $|M|$. Moreover It is clear that all rational normal scrolls $X \in \mathbb{F}_{\underline{a}}$ are projectively equivalent in $\mathbb{P}^{n}$.

Therefore fixing a morphism $\bar{g}_{0}: \mathbb{F}(\underline{a}) \rightarrow \mathbb{P}^{n}$, the family $\mathcal{F}_{\underline{a}}$ is isomorphic to the quotient $\operatorname{PGL}(n+1, \mathbb{K}) /$ Aut, where the group Aut is an algebraic subgroup of $\operatorname{PGL}(n+1, \mathbb{K})$ fixing the scroll $g_{0}(\mathbb{F}(\underline{a}))$. In particular $\mathcal{F}_{\underline{a}}$ is an irreducible quasi projective variety.

To complete the proof we must evaluate the dimension of Aut. With this aim we will show that any automorphism of $\mathbb{F}(\underline{a})$ can be lifted to an element of $\operatorname{PGL}(n+1, \mathbb{K})$, i.e. Aut $\cong \operatorname{Aut}(\mathbb{F}(\underline{a}))$, and we will estimate $\operatorname{dim} \operatorname{Aut}(\mathbb{F}(\underline{a}))$.

Since $\mathbb{F}(\underline{a}) \not \mathbb{F}(a, a)=\mathbb{P}^{1} \times \mathbb{P}^{1}$ there is a unique projection map $\mathbb{F}(\underline{a}) \rightarrow \mathbb{P}^{1}$, thus any automorphism on $\mathbb{F}(\underline{a})$ induces an automorphism on $\mathbb{P}^{1}$. It is clear that the converse holds: any automorphism on $\mathbb{P}^{1}$ can be lifted to an automorphism on $\mathbb{F}(\underline{a})$ and to an automorphism in Aut.

Consider the following exact sequence

$$
0 \rightarrow \operatorname{Aut}_{0} \rightarrow \operatorname{Aut}(\mathbb{F}(\underline{a})) \rightarrow \operatorname{Aut}\left(\mathbb{P}^{1}\right) \rightarrow 0
$$

where $\mathrm{Aut}_{0}$ is the subgroup of automorphisms of $\mathbb{F}(\underline{a})$ commuting with the projection to $\mathbb{P}^{1}$. It is immediately seen that $\operatorname{Aut}_{0} \cong \mathbb{P}(\operatorname{Aut}(\underline{a}))=\mathbb{P}\left(\operatorname{Aut}\left(\bigoplus_{i=1}^{d} \mathcal{O}_{\mathbb{P}^{1}}\left(a_{i}\right)\right)\right)$ 
(see [11, Proposition II.7.12]) and every automorphism can be lifted to an element of $\operatorname{PGL}(n+1, \mathbb{K})$. A simple induction argument shows that

$$
\operatorname{dim} \operatorname{Aut}(\underline{a}) \geq d^{2}
$$

and equality holds if and only if $\left|a_{i}-a_{1}\right| \leq 1$ for every $i$.

We conclude that

$$
\begin{aligned}
\operatorname{dim} \mathcal{F}_{\underline{a}} & =\left((n+1)^{2}-1\right)-\left(\operatorname{dim} \operatorname{Aut}\left(\bigoplus_{i=1}^{d} \mathcal{O}_{\mathbb{P}^{1}}\left(a_{i}\right)\right)-1\right)-3 \\
& =n^{2}+2 n-2-d^{2}-\left(\operatorname{dim} \operatorname{Aut}\left(\bigoplus_{i=1}^{d} \mathcal{O}_{\mathbb{P}^{1}}\left(a_{i}\right)\right)-d^{2}\right)
\end{aligned}
$$

This exactly means that

$$
\operatorname{dim} \mathcal{F}_{\underline{a}} \leq n^{2}+2 n-2-d^{2} .
$$

with equality holding if and only if $\left|a_{i}-a_{1}\right| \leq 1$ for every $i$.

It remains the case $\underline{a}=\{a, a\}$. In this case we need to take into account also the automorphism of $\mathbb{F}(a, a) \cong \mathbb{P}^{1} \times \mathbb{P}^{1}$ swapping the two $\mathbb{P}^{1}$ 's. See [2] for the details.

The dimension of the family $\mathcal{G}$ of rational normal curves is just a particular case of the case of rational normal scrolls. See for example [4].

Finally, $\mathcal{F}(S)_{\underline{a}}$ is isomorphic to the family $U / \operatorname{Aut}\left(\mathbb{F}\left(a_{1}, \ldots, a_{d}\right)\right)$, where $U$ is the open subscheme of $\mathbb{F}\left(a_{1}, \ldots, a_{d}\right)^{n+2}$ given by $n+2$ points imposing independent conditions on $|M|$. Therefore

$$
\operatorname{dim} \mathcal{F}(S)_{\underline{a}}=\operatorname{dim} \mathbb{F}\left(a_{1}, \ldots, a_{d}\right)^{n+2}-\operatorname{dim} \operatorname{Aut}\left(\mathbb{F}\left(a_{1}, \ldots, a_{d}\right)\right)
$$

and we may conclude.

Proposition 3.2 The family $\mathcal{F}_{0}$ of balanced scrolls is dense in the family $\mathcal{F}$ of rational normal scrolls of dimension $d$ and degree $n-d+1$ in $\mathbb{P}^{n}$.

Proof. (See [13, chapter 2,Exercises 22, 23]).

Consider a multi-index $\underline{a}=\left(a_{1}, \ldots, a_{d}\right)$, and assume that $a_{i} \geq 0$ for every $i$ and $a_{1} \geq 1$. We do not need to assume that $a_{1} \leq a_{2} \leq \ldots \leq a_{d}$. We will prove that there is a family of scrolls in $\mathbb{P}^{n}$, each of them image of $\mathbb{F}\left(a_{1}, \ldots, a_{d}\right)$, which degenerates to a scroll which is image of $\mathbb{F}\left(a_{1}-1, a_{2}, \ldots, a_{d-1}, a_{d}+1\right)$. This is clearly enough to conclude the statement.

Consider at first the $(d+1)$-dimensional scroll

$$
\mathbb{F}^{d+1}=\mathbb{F}\left(a_{1}-1, a_{2}, \ldots, a_{d-1}, a_{d}, 1\right) \rightarrow \mathbb{P}^{n+1}
$$


and let $M^{\prime}$ be the relative hyperplane divisor. The scroll $\mathbb{F}\left(a_{1}, \ldots, a_{d}\right)$ belongs to the linear system $\left|M^{\prime}\right|$ in $\mathbb{F}^{d+1}$ since we have the embedding

$$
\begin{array}{rlrl}
\varphi_{1}: & \mathbb{F}\left(a_{1}, \ldots, a_{d}\right) & \hookrightarrow & \mathbb{F}\left(a_{1}-1, a_{2}, \ldots, a_{d-1}, a_{d}, 1\right) \\
\left(x_{1}, \ldots, x_{d}\right) \times\left(t_{0}, t_{1}\right) & \mapsto & \left(t_{0} x_{1}, x_{2}, \ldots, x_{d}, t_{1}^{a_{1}-1} x_{1}\right) \times\left(t_{0}, t_{1}\right)
\end{array}
$$

Note that $\operatorname{Im}\left(\varphi_{1}\right)=\left\{t_{0} y_{d+1}=t_{1}^{a_{1}-1} y_{1}\right\} \subset \mathbb{F}^{d+1}$.

Similarly we have a second embedding

$$
\begin{array}{cccc}
\varphi_{2}: \mathbb{F}\left(a_{1}-1, a_{2}, \ldots, a_{d-1}, a_{d}+1\right) & \hookrightarrow & \mathbb{F}\left(a_{1}-1, a_{2}, \ldots, a_{d-1}, a_{d}, 1\right) \\
\left(x_{1}, \ldots, x_{d}\right) \times\left(t_{0}, t_{1}\right) & \mapsto & \left(x_{1}, x_{2}, \ldots, t_{0} x_{d}, t_{1}^{a_{d}} x_{d}\right) \times\left(t_{0}, t_{1}\right)
\end{array}
$$

It is clear that $\operatorname{Im}\left(\varphi_{2}\right)=\left\{t_{0} y_{d+1}=t_{1}^{a_{d}} y_{d}\right\} \subset \mathbb{F}^{d+1}$.

Consider now the family of divisors in $\left|M^{\prime}\right|$ given by the equation

$$
\left\{t_{0} y_{d+1}=\lambda t_{1}^{a_{1}-1} y_{1}+t_{1}^{a_{d}} y_{d}\right\} \text { for } \lambda \in \mathbb{C} .
$$

For $\lambda \neq 0$ we have a family of scrolls isomorphic to $\operatorname{Im}\left(\varphi_{1}\right)$, which degenerates to $\operatorname{Im}\left(\varphi_{2}\right)$ when $\lambda=0$.

\section{Rational normal curves contained in scrolls}

First of all let us recall the following characterisation of rational normal curves contained in a scroll.

Remark 4.1 Fix a multi-index $\underline{a}=\left\{a_{1}, \ldots, a_{d}\right\}$, consider a scroll $\mathbb{F}\left(a_{1}, \ldots, a_{d}\right)$, and the associated morphism: $\varphi: \mathbb{F}\left(a_{1}, \ldots, a_{d}\right) \rightarrow \mathbb{P}^{n}$.

Let $\mathbb{F}:=\varphi\left(\mathbb{F}\left(a_{1}, \ldots, a_{d}\right)\right)$. By the birationality of $\varphi$, a rational normal curve $\Gamma \subset \mathbb{F}$ corresponds to a unique embedding $\psi: \Gamma \hookrightarrow \mathbb{F}\left(a_{1}, \ldots, a_{d}\right)$ which can be explicitly written with respects to the coordinates $\left(s_{0}, s_{1}\right)$ on $\Gamma$ :

$$
\begin{array}{cccc}
\psi: \quad \begin{array}{c}
\Gamma \\
\left(s_{0}, s_{1}\right)
\end{array} & \mapsto & \mathbb{F}\left(a_{1}, \ldots, a_{d}\right) \\
& \left.\mapsto y_{1}\left(s_{0}, s_{1}\right), \ldots, y_{d}\left(s_{0}, s_{1}\right)\right) \times\left(t_{0}\left(s_{0}, s_{1}\right), t_{1}\left(s_{0}, s_{1}\right)\right)
\end{array}
$$

The induced map $\Gamma \rightarrow \mathbb{P}^{1}$ is the composition:

$$
\begin{array}{ccc}
\Gamma & \rightarrow & \mathbb{P}^{1} \\
\left(s_{0}, s_{1}\right) & \mapsto & \left(t_{0}\left(s_{0}, s_{1}\right), t_{1}\left(s_{0}, s_{1}\right)\right)
\end{array}
$$

whose degree is $\operatorname{deg} t_{0}\left(s_{0}, s_{1}\right)=\operatorname{deg} t_{1}\left(s_{0}, s_{1}\right)=k$.

Since $\Gamma$ is a rational normal curve in $\mathbb{P}^{n}$ we have that $\operatorname{deg}\left(\mathcal{O}_{\mathbb{P}^{n}}(1)_{\mid \Gamma}\right)=\Gamma \cdot M=$ $n$. The divisor $\left(y_{i}=0\right) \subset \mathbb{F}\left(a_{1}, \ldots, a_{d}\right)$ is in the linear system $\left|M-a_{i} L\right|$, hence $\operatorname{deg} y_{i}\left(s_{0}, s_{1}\right)=n-a_{i} k$.

We are going to study family of rational normal curves contained in a fixed scroll and conversely study rational normal scrolls containing some rational curves. We start with the following obvious remark. 
Remark 4.2 Let $\mathbb{F}$ be a rational normal scroll of minimal degree in $\mathbb{P}^{n}$ and let $P_{0} \in \mathbb{F}$. Consider the projection map from $P_{0}$ onto a general hyperplane

$$
\pi_{P_{0}}: \mathbb{P}^{n} \longrightarrow \mathbb{P}^{n-1} .
$$

Then $\overline{\pi_{P_{0}}(\mathbb{F})}$ is still a rational scroll of minimal degree in $\mathbb{P}^{n-1}$. Moreover if $P_{0}$ is smooth then $\operatorname{dim}\left(\overline{\pi_{P_{0}}(\mathbb{F})}\right)=\operatorname{dim}(\mathbb{F})$, whereas if $P_{0}$ is singular then $\operatorname{dim}\left(\overline{\pi_{P_{0}}(\mathbb{F})}\right)=\operatorname{dim}(\mathbb{F})-1$.

Our analysis is subdivided in several steps.

Proposition 4.3 Let $\mathbb{F}\left(a_{1}, \ldots, a_{d}\right)$ be a scroll of dimension $d$ and let $\mathbb{F}$ be its image in $\mathbb{P}^{n}$.

Let $\mathcal{G}^{\vee}(\mathbb{F}, k)$ be the family of rational normal curves $\Gamma$ in $\mathbb{F}$ such that the induced map $\Gamma \rightarrow \mathbb{P}^{1}$ has degree $k$. Then

- $\mathcal{G}^{\vee}(\mathbb{F}, k)$ is irreducible and has dimension $(d-1)(n+3-k)+(k-1)(2 d-n)$ if $k \leq d$ and $n-a_{i} k \geq 0$ for every $i$;

- $\mathcal{G}^{\vee}(\mathbb{F}, k)=\emptyset$ otherwise.

Proof. Notice that there can not be a curve $\Gamma$ with $k>d$ since the general fibre of $\Gamma \rightarrow \mathbb{P}^{1}$ consists of $k$ points in linear general position, contained in a fibre of $\mathbb{F}\left(a_{1}, \ldots, a_{d}\right)$, which is a $(d-1)$-dimensional projective subspace of $\mathbb{P}^{n}$. Therefore from now on we may assume that $k \leq d$.

We have seen in Remark 4.1 that a rational normal curve $\Gamma \subset \mathbb{F}$ is determined by its coordinates

$$
\begin{aligned}
& \psi: \quad \Gamma \quad \rightarrow \quad \mathbb{F}\left(a_{1}, \ldots, a_{d}\right) \\
& \left(s_{0}, s_{1}\right) \mapsto\left(y_{1}\left(s_{0}, s_{1}\right), \ldots, y_{d}\left(s_{0}, s_{1}\right)\right) \times\left(t_{0}\left(s_{0}, s_{1}\right), t_{1}\left(s_{0}, s_{1}\right)\right)
\end{aligned}
$$

Such polynomials can not exist if $\operatorname{deg} y_{i}=n-k a_{i}<0$ for some $i$, hence in this case there are no rational curves with the required properties. On the contrary if $\operatorname{deg} y_{i}=n-k a_{i} \geq 0$ for every $i$ then the family is nonempty.

It is clear that the coordinates $\left\{y_{1}, \cdots, y_{d}, t_{0}, t_{1}\right\}$ are unique up to the action of $\operatorname{Aut}(\Gamma)$ and of $\left(\mathbb{C}^{*}\right)^{2}$ on $\mathbb{F}\left(a_{1}, \ldots, a_{d}\right)$.Therefore we get

$$
\begin{aligned}
\operatorname{dim} & =\sum_{i=1}^{d} h^{0}\left(\mathbb{P}^{1}, \mathcal{O}_{\mathbb{P}^{1}}\left(n-k a_{i}\right)\right)+2 h^{0}\left(\mathbb{P}^{1}, \mathcal{O}_{\mathbb{P}^{1}}(k)\right)-3-2 \\
& =(d-1)(n+3-k)+(k-1)(2 d-n)
\end{aligned}
$$

This family is clearly irreducible, since it is dominated by an open set of a vector space.

Now we analyse the simplest cases of rational normal scrolls: hypersurface scrolls, i.e., quadrics of rank 3 or 4 . 
Lemma 4.4 Let $Q=\left\{q\left(x_{0}, \ldots, x_{n}\right)=0\right\}$ be a quadric of rank 3 or 4 in $\mathbb{P}^{n}$. Let $S \subset Q$ be a set of $n+2$ points in linear general position in $\mathbb{P}^{n}$ and assume that

$$
S \cap \operatorname{Sing}(Q)=\emptyset .
$$

Then there exists at most a finite number of rational normal curves $\Gamma$ such that $S \subset \Gamma \subset Q$.

Proof. Assume that $S$ is the set of coordinate points $(0: \ldots: 0: 1: 0: \ldots, 0)$ in $\mathbb{P}^{n}$ and the point $(1: \ldots: 1)$.

It is well known that, up to Aut $\left(\mathbb{P}^{1}\right)$, every rational normal curve $\Gamma$ through $S$ is given by the map

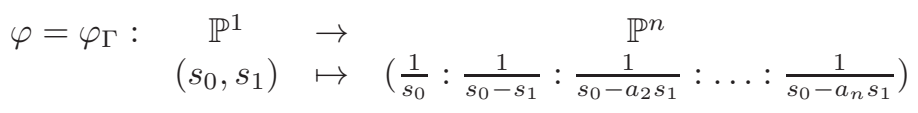

and that the numbers $a_{i}$ determine uniquely the curve. Since $S \subset Q$ we have that

$$
q\left(\varphi_{\Gamma}\left(s_{0}, s_{1}\right)\right)=s_{0} s_{1}\left(s_{0}-s_{1}\right) \prod_{i=2}^{n}\left(s_{0}-a_{i} s_{1}\right) \cdot p\left(s_{0}, s_{1}\right)
$$

where $p\left(s_{0}, s_{1}\right)$ is a degree $n-2$ polynomial in $\left(s_{0}, s_{1}\right)$ with coefficients depending on the parameters $a_{i}$.

The rational normal curves we are looking for correspond to parameters $\left(a_{2}, \cdots, a_{n}\right)$ such that $p\left(s_{0}, s_{1}\right) \equiv 0$ on $\mathbb{P}^{1}$, where $p$ is defined in equation (2) and it depends on $\left(a_{2}, \cdots a_{n}\right)$. A simple Bertini's type argument shows that for a general choice of the parameters $a_{2}, \cdots, a_{n}$ the polynomial $q\left(\varphi\left(s_{0}, s_{1}\right)\right)$ has $2 n$ distinct zeroes.Notice that the assumption $S \cap \operatorname{Sing}(Q)=\emptyset$ is necessary since otherwise for every $\Gamma$ the polynomial $q\left(\varphi_{\Gamma}\left(s_{0}, s_{1}\right)\right)$ should vanish with order $\geq 2$ at the preimage of every point in $S \cap \operatorname{Sing}(Q)$.

Consider now $n-1$ general points in in $\mathbb{P}^{1}: T_{1}, \ldots, T_{n-1}$. Let $\mathcal{G}_{0}=\mathcal{G}(S)$ be the family of rational normal curves in $\mathcal{G}$ containing $S$ and for every $j=$ $1, \cdots n-1$ define inductively $\mathcal{G}_{j}$ to be the closure in $\mathcal{G}$ of the family of rational normal curves $\Gamma$ through $S$ such that $\varphi_{\Gamma}\left(T_{1}\right), \ldots, \varphi_{\Gamma}\left(T_{j}\right) \in Q \backslash S$. It is clear that $\mathcal{G}_{j}$ corresponds to the family of parameters $\left\{a_{2}, \ldots, a_{n}\right\}$ such that

$$
p\left(T_{1}\right)=\ldots=p\left(T_{j}\right)=0 .
$$

Notice that $\mathcal{G}_{n-1}$ is the family of rational normal curves contained in $Q$ since $\operatorname{deg}(p)=n-2$. Now it is $\operatorname{dim} \mathcal{G}_{0}=n-1$ by Theorem 3.1. Therefore the lemma follows if we show that

$$
\operatorname{dim} \mathcal{G}_{j+1}=\operatorname{dim} \mathcal{G}_{j}-1 .
$$

Consider the incidence correspondence

$$
I=\left\{(\Gamma, x): \varphi_{\Gamma}(x) \in Q \backslash S\right\} \subset \mathcal{G}_{j} \times \mathbb{P}^{1} \backslash\left\{T_{1}, \ldots, T_{j}\right\}
$$


The map $I \rightarrow \mathcal{G}_{j}$ is generically finite thanks to the existence of generic map $\varphi$ we discuss above, thus $\operatorname{dim} I=\operatorname{dim} X_{j}$. Moreover the map $I \rightarrow \mathbb{P}^{1} \backslash\left\{T_{1}, \ldots, T_{j}\right\}$ is nonconstant by genericity of $\varphi$, thus the general fibre has codimension 1 . Since the general fibre over $P_{j+1}$ is $\mathcal{G}_{j+1}$ we conclude.

Lemma 4.5 Let $Q=\left\{q\left(x_{0}, \ldots, x_{n}\right)=0\right\}$ be a quadric of rank 3 or 4 in $\mathbb{P}^{n}$. Let $S \subset Q$ be a set of $n+2$ points in linear general position in $\mathbb{P}^{n}$. Then the family of rational normal curves $\Gamma$ such that $S \subset \Gamma \subset Q$ has dimension at most $\#(S \cap \operatorname{Sing}(Q))$.

Proof. It is a simple induction $\operatorname{argument}$ on $\#(S \cap \operatorname{Sing}(Q))$. If $\#(S \cap \operatorname{Sing}(Q))=$ 0 we are back to the previous Lemma. Assume that $\#(S \cap \operatorname{Sing}(Q)) \neq 0$.

Consider one point $P \in S \cap \operatorname{Sing}(Q)$ and project away from it, $\pi_{P}: \mathbb{P}^{n} \rightarrow$ $\mathbb{P}^{n-1}$. The degree 2 polynomial $q$ does not change via the projection since it is singular at $P$. Then the family of rational normal curves $\Gamma$ such that $S \subset \Gamma \subset Q$ is obviously given by all the possible liftings through $P$ of the rational normal curves $\Gamma \subset \mathbb{P}^{n-1}$ such that $\pi_{P}(S \backslash P) \subset \Gamma \subset(q=0)$. Since every curve in $\mathbb{P}^{n-1}$ has a one dimensional family of liftings, we conclude by induction.

Now we give an estimate of the dimension of the family of rational normal curves passing through $n+2$ general points and contained in a fixed rational normal scroll.

Proposition 4.6 Let $\mathbb{F}$ be a rational normal scroll of dimension d and minimal degree in $\mathbb{P}^{n}$, with $n \geq d+1$. Let $S \subset \mathbb{F}$ a set of $n+2$ points in linear general position in $\mathbb{P}^{n}$. Then the family of rational normal curves $\Gamma$ such that $S \subset \Gamma \subset \mathbb{F}$ has dimension at most \# $(S \cap \operatorname{Sing}(\mathbb{F}))$.

Proof. We argue by induction on $n-d$. If $n-d=1$ then $\mathbb{F}$ is a quadric of rank 3 or 4, and we apply the previous Lemma.

Assume now $n-d>1$.

If $S \cap \operatorname{Sing}(\mathbb{F}) \neq \emptyset$ we use the same projection argument adopted in the previous Lemma, and we conclude by induction.

Thus we may assume that $S \cap \operatorname{Sing}(\mathbb{F})=\emptyset$.

Take $P \in S$ and consider the projection $\pi_{P}: \mathbb{P}^{n} \rightarrow \mathbb{P}^{n-1}$.

By Remark 4.2 the closure of $\pi_{P}(\mathbb{F})$ is a rational normal scroll $\mathbb{F}_{0}$ of dimension $d$ and minimal degree in $\mathbb{P}^{n-1}$.

Every rational normal curve $\Gamma$ in $\mathbb{F}$ through $S$ projects to a rational normal curve $\Gamma_{0}$ in $\mathbb{F}_{0}$ passing through the image of the remaining $n+1$ points in $\mathbb{P}^{n-1}$. Moreover it is easy to check that none of these $n+1$ points is in the singular locus of $\mathbb{F}_{0}$, thus by induction we may assume that there is at most a finite number of rational normal curves through them.

A rational normal curve $\Gamma_{0}$ in the scroll $\mathbb{F}_{0} \subset \mathbb{P}^{n-1}$ has precisely one lifting in $\mathbb{F}$ by birationality, thus it has at most one lifting which passes through $P$. Therefore, by induction, we may conclude. 
Lemma 4.7 Let $\mathbb{F}=\mathbb{F}\left(a_{1}, \ldots, a_{d}\right)$ be a rational normal scroll, $\pi: \mathbb{F}\left(a_{1}, \ldots, a_{d}\right) \rightarrow$ $\mathbb{P}^{1}$ be the usual projection, and let $L=\pi^{*}\left(\mathcal{O}_{\mathbb{P}^{1}}(1)\right)$ and $M$ a hyperplane divisor.

Let $S$ be a set of $n+2$ distinct points imposing independent conditions on the linear system $|M|$. Then $\pi_{\mid S}: S \rightarrow \mathbb{P}^{1}$ is injective if and only if there exists a smooth rational curve $\Gamma$ such that

1. $S \subset \Gamma \subset \mathbb{F}\left(a_{1}, \ldots, a_{d}\right)$;

2. $\Gamma \cdot L=1$;

3. $\Gamma \cdot M=n$.

Moreover if such a $\Gamma$ exists it is unique.

Proof. Assume there exists a rational normal curve $\Gamma$ satisfying the conditions 1., 2., 3. Since $\Gamma \cdot L=1$ we have that $\pi$ is an isomorphism on $\Gamma$, thus it must be injective on $S \subset \Gamma$ too.

Assume now that $\pi_{\mid S}$ is injective. We are going to prove that the intersection of every divisor in $|L+M|$ containing $S$ is indeed a smooth rational curve $\Gamma$ satisfying 1., 2., 3 .

We will prove it by induction on $d=\operatorname{dim} \mathbb{F}$.

If $d=1$ the statement is trivial.

Assume now $d \geq 2$. The system $|L+M|$ is base point free of projective dimension $n+d$, hence the restriction map $H^{0}(\mathbb{F}, L+M) \rightarrow H^{0}\left(S, \mathcal{O}_{S}\right)$ has nontrivial kernel, since $h^{0}\left(S, \mathcal{O}_{S}\right)=n+2$. Thus there exists a divisor $D \in$ $|L+M|$ containing $S . D$ is a $(d-1)$-dimensional scheme and we may assume $D$ integral. Indeed, assuming by a contradiction that $D$ is not integral then there should exist a positive integer $e$ so that we can write $D=D_{1}+D_{2}$, with $D_{1} \in|e L|$ and $D_{2} \in|M-e L|$ (cf. [13, Chapter 2]). But $h^{0}\left(X, D_{1}\right) \leq e+1$ and $h^{0}\left(X, D_{2}\right) \leq n-e+1$, i.e. at most $e$ point in $S$ can be contained in the divisor $D_{1}$ since $\pi$ is injective on $S$ and at most $n-e$ points in $S$ can be contained in the divisor $D_{2}$ since $S$ imposes independent conditions on $|M|$, which is absurd.

Therefore by construction $D$ is a rational normal scroll of dimension $d-1$ containing $S$, whose projection $\pi: D \rightarrow \mathbb{P}^{1}$ is the restriction of the projection of $\mathbb{F}$. Notice that $\operatorname{deg}\left(M_{\mid D}^{d-1}\right)=D \cdot M^{d-1}=(L+M) \cdot M^{d-1}=n-d+2$. Thus we may apply the induction argument to $D$, endowed with the line bundles $L_{\mid D}, M_{\mid D}$. By induction we know that the intersection of every divisor in $H^{0}\left(D, L_{\mid D}+M_{\mid D}\right)$ containing $S$ is a smooth rational curve $\Gamma$, and the intersection of every divisor in $H^{0}(\mathbb{F}, L+M)$ containing $S$ must coincide with it.

To conclude the statement we need to prove that such smooth rational curve, if it exists, is unique. Assume that $\pi_{\mid S}$ is injective. If $\Gamma_{0}$ is any smooth rational curve satisfying the conditions $1,2,3$ we have that the restriction map $H^{0}\left(\Gamma_{0}, L+M\right)=H^{0}\left(\Gamma_{0}, \mathcal{O}_{\Gamma_{0}}(1+n)\right) \rightarrow H^{0}\left(S, \mathcal{O}_{S}\right)$ is an isomorphism, since $\Gamma_{0} \cong \mathbb{P}^{1}$. Thus $H^{0}\left(\mathbb{F}, \mathcal{I}_{\Gamma_{0}}(L+M)\right)=H^{0}\left(\mathbb{F}, \mathcal{I}_{S}(L+M)\right)$, hence $\Gamma_{0}$ is contained in the intersection of the sections of $H^{0}(\mathbb{F}, L+M)$ vanishing on $S$. But we have just proved that this intersection is precisely the smooth rational curve $\Gamma$, thus $\Gamma_{0}=\Gamma$. 
Proposition 4.8 Assume $n \geq 2 d$ and let $\mathbb{F}$ be a rational normal scroll of dimension d in $\mathbb{P}^{n}$. Consider a set $S \subset \mathbb{F}$ of $n+2$ points in linear general position.

Then the family of rational normal curves $\Gamma$ such that $S \subset \Gamma \subset \mathbb{F}$ and the induced projection $\Gamma \rightarrow \mathbb{P}^{1}$ has degree $k$, with $1 \leq k \leq d$, is either empty, or it has positive dimension, or it consists of one single curve.

Proof. Let $\mathcal{G}(S)$ be the family of rational normal curves containing $S$ and let $\mathcal{G}^{\vee}(\mathbb{F}, k)$ be the family of rational normal curves $\Gamma$ in $\mathbb{F}$ such that the induced map $\Gamma \rightarrow \mathbb{P}^{1}$ has degree $k$. We are going to study the family $\mathcal{G}^{\vee}(\mathbb{F}, k) \cap \mathcal{G}(S)$.

If $k=1$ we apply Lemma 4.7. Consider the map $\mathbb{F}\left(a_{1}, \ldots, a_{d}\right) \rightarrow \mathbb{F}$. If there exists a rational normal curve $\Gamma$ with the required properties, then $S$ and $\Gamma$ can be lifted to a set of $n+2$ points and a smooth rational curve in $\mathbb{F}\left(a_{1}, \ldots, a_{d}\right)$ as described in Lemma 4.7. In particular, it is clear that the family of suitable $\Gamma$ 's coincides with the family of liftings of $S$ in $\mathbb{F}\left(a_{1}, \ldots, a_{d}\right)$. If $S \cap \operatorname{Sing}(\mathbb{F})=\emptyset$ the lifting is unique and there exists at most one rational normal curve. Otherwise there is a positive dimensional family of liftings.

Assume now $k \geq 2$. We work by induction on $n-2 d$. Assume at first that $n-2 d=0$.

Consider the incidence correspondence

$$
I_{k}=\left\{\left(\Gamma, P_{1}, \ldots, P_{n+2}\right): P_{i} \in \Gamma\right\} \subset \mathcal{G}^{\vee}(\mathbb{F}, k) \times \mathbb{F}\left(a_{1}, \ldots, a_{d}\right)^{n+2} .
$$

Proposition 4.3 implies that $\operatorname{dim} I_{k}=(d-1)(n+3-k)+(n+2)$ since the projection $I_{k} \rightarrow \mathcal{G}^{\vee}(\mathbb{F}, k)$ is surjective with $(n+2)$-dimensional fibres.

Consider now the map $I_{k} \rightarrow \mathbb{F}\left(a_{1}, \ldots, a_{d}\right)^{n+2}$. It is generically injective, i.e., there exists $S \in \mathbb{F}\left(a_{1}, \ldots, a_{d}\right)^{n+2}$ having precisely one preimage in $I_{k}$. Indeed, by the polynomial description of the curves we made in Proposition 4.3 , and a dimensional computation, a point in $\mathbb{F}$ imposes $d-1$ independent conditions on the family of the curves with degree $k$ map to $\mathbb{P}^{1}$. Similarly, $n+3-k$ general points in $\mathbb{F}$ impose precisely $(d-1)(n+3-k)$ conditions on such family, thus by Proposition 4.3 there exists a finite number of suitable rational normal curves through those points. By fixing one of them and taking other $k-1$ points on it we obtain the length $n+2$ scheme $S$ we were looking for.

The map $I_{k} \rightarrow \mathbb{F}\left(a_{1}, \ldots, a_{d}\right)^{n+2}$, is a quasi-projective morphism since $I_{k} \subset$ $\mathcal{G} \times \mathbb{F}\left(a_{1}, \ldots, a_{d}\right)^{n+2}$ and $\mathcal{G}$ is quasi-projective.

Since there is a point in $\mathbb{F}\left(a_{1}, \ldots, a_{d}\right)^{n+2}$ with precisely one preimage, by a Stein factorisation argument (see [11, Corollary III.11.5]) we see that every fibre is connected. In particular every set of $n+2$ points with zero dimensional fibre (in particular those points avoiding the singular locus of $\mathbb{F}$ by Proposition 4.6) must have precisely one preimage, proving our statement.

Assume now that $n>2 d$. Fix $S \subset \mathbb{F}$ and assume that there is a finite number of rational normal curve $\Gamma$ such that $S \subset \Gamma \subset \mathbb{F}$ and the induced projection $\Gamma \rightarrow \mathbb{P}^{1}$ has degree $k$. With the usual projection argument used in Proposition 4.6 we may assume that $S \cap \operatorname{Sing}(\mathbb{F})=\emptyset$.

Consider a point $P \in S$ and the projection $\pi_{P}$ onto $\mathbb{P}^{n-1}$. 
By Proposition 4.6 and induction there is exactly one rational normal curve $\Gamma_{0}$ in $\mathbb{P}^{n-1}$ such that $\pi(S \backslash\{P\}) \subset \Gamma_{0} \subset \pi_{P}(\mathbb{F})$.

But a rational normal curve in the scroll $\pi_{P}(\mathbb{F}) \subset \mathbb{P}^{n-1}$ has one lifting in $\mathbb{F}$ by birationality, thus it has at most one lifting which passes through $P$, and we may conclude by induction.

Finally we are going to consider families of scrolls which contains a rational normal curve $\Gamma$ passing through $n+2$ fixed points, such that the induced map $\Gamma \rightarrow \mathbb{P}^{1}$ has degree $k$.

Proposition 4.9 Assume that $n$ is an even number and let $d=\frac{n}{2}$. Let $\underline{a}=$ $\left\{a_{1}, \ldots, a_{d}\right\}$ be such that $\sum a_{i}=n-d+1$.

Consider a set $S$ of $n+2$ points in linear general position in $\mathbb{P}^{n}$ and let $\mathcal{F}(S, k)_{\underline{a}}$ be the family consisting of $\mathbb{F}$ isomorphic to $\mathbb{F}(\underline{a})$ such that there exists a rational normal curve $\Gamma$ so that $S \subset \Gamma \subset \mathbb{F}$ and the induced map $\Gamma \rightarrow \mathbb{P}^{1}$ has degree $k$. Then

- if $n-k a_{i} \geq 0$ for every $i$ we have

$$
\begin{aligned}
\operatorname{dim} \mathcal{F}(k)_{\underline{a}} & =\frac{n^{2}}{4}+n-2-(k-1)\left(\frac{n}{2}-1\right)-\left(\operatorname{dim} \operatorname{Aut}(\underline{a})-\frac{n^{2}}{4}\right) \\
& =\operatorname{dim} \mathcal{F}(S)_{\underline{a}}-(k-1)\left(\frac{n}{2}-1\right)
\end{aligned}
$$

- otherwise $\mathcal{F}(k)_{\underline{a}}$ is empty.

Proof. If $n-k a_{i}<0$ for some $i$ Proposition 4.3 tells us that there are no rational normal curves with the required degree on $\mathbb{P}^{1}$, thus $\mathcal{F}(k)_{a}$ is empty. We may therefore assume that this is not the case.

In Thm. 3.1 we have seen that $\mathcal{F}(S)_{\underline{a}}$ is isomorphic to an open subset of $\mathbb{F}\left(a_{1}, \ldots, a_{d}\right)^{n+2} / \operatorname{Aut}\left(\mathbb{F}\left(a_{1}, \ldots, a_{d}\right)\right)$. According to this isomorphism the family we are looking for, $\mathcal{F}(k)_{\underline{a}}$, coincides with the set of $n+2$ points in $\mathbb{F}\left(a_{1}, \ldots, a_{d}\right)$ contained in some rational normal curve $\Gamma$, such that $\operatorname{deg}\left(\Gamma \rightarrow \mathbb{P}^{1}\right)=k$ (up to $\left.\operatorname{Aut}\left(\mathbb{F}\left(a_{1}, \ldots, a_{d}\right)\right)\right)$. We have seen how these curve are characterised in Proposition 4.3

Consider the incidence correspondence

$$
I_{k}=\left\{\left(\Gamma, P_{1}, \ldots, P_{n+2}\right): P_{i} \in \Gamma\right\} \subset \mathcal{G}^{\vee}(\mathbb{F}(\underline{a}), k) \times \mathbb{F}(\underline{a})^{n+2} .
$$

Thanks to Proposition 4.3 we know that $\operatorname{dim} I_{k}=(d-1)(n+3-k)+n+2$. Consider the projection $\varphi: I_{k} \rightarrow \mathbb{F}\left(a_{1}, \ldots, a_{d}\right)^{n+2}$. Since the space $\mathcal{F}(k)_{a}$ is $\varphi\left(I_{k}\right) / \operatorname{Aut}\left(\mathbb{F}\left(a_{1}, \ldots, a_{d}\right)\right)$, its dimension is at most $\operatorname{dim} I_{k}-\operatorname{dim} \operatorname{Aut}\left(\mathbb{F}\left(a_{1}, \ldots, a_{d}\right)\right)$ :

$$
\operatorname{dim} \mathcal{F}(k)_{\underline{a}} \leq \frac{n^{2}}{4}+n-2-(k-1)\left(\frac{n}{2}-1\right)-\left(\operatorname{dim} \operatorname{Aut}(\underline{a})-\frac{n^{2}}{4}\right) .
$$

To prove the opposite inequality we argue as in the proof of Lemma 4.8 . Consider the characterisation of rational normal curves as a set of polynomials 
of prescribed degree, up to automorphism, given in Proposition 4.3. A simple linear algebra computation shows that passing through a fixed point imposes at most $d-1=\frac{n}{2}-1$ conditions on those polynomials, thus for $n+3-k$ general points in $\mathbb{F}\left(a_{1}, \ldots, a_{d}\right)$ there exists at least one rational normal curve with the right degree. Fixing $n+3-k$ general points, we can moreover choose the remaining $k-1$ points on this curve. Thus we find a space of dimension $d \cdot(n+3-k)+(k-1)$ in $\mathbb{F}\left(a_{1}, \ldots, a_{d}\right)^{n+2}$ which is in the image of the incidence correspondence $I \rightarrow \mathbb{F}\left(a_{1}, \ldots, a_{d}\right)^{n+2}$. Therefore $\mathcal{F}(k)_{\underline{a}}$ has dimension at least $d \cdot(n+3-k)+(k-1)-\operatorname{dim} \operatorname{Aut}\left(\mathbb{F}\left(a_{1}, \ldots, a_{d}\right)\right)$, or equivalently

$$
\operatorname{dim} \mathcal{F}(k)_{\underline{a}} \geq \frac{n^{2}}{4}+n-2-(k-1)\left(\frac{n}{2}-1\right)-\left(\operatorname{dim} \operatorname{Aut}(\underline{a})-\frac{n^{2}}{4}\right) .
$$

Proposition 4.10 Let $S$ be a set of $n+2$ points in linear general position in $\mathbb{P}^{n}$ and let $\mathcal{F}(S, k)_{\underline{a}}$ and $\mathcal{F}(S, h)_{\underline{a}}$ be as in Proposition 4.9 . Assume that $k \neq h$ and $k+h \geq \frac{n}{2}+1$. Then

$$
\operatorname{dim}\left(\mathcal{F}(S, k)_{\underline{a}} \cap \mathcal{F}(S, h)_{\underline{a}}\right) \leq 2 n-3 .
$$

Proof. By Proposition $4.9 \mathcal{F}(S)_{\underline{a}}$ is isomorphic to an open subset

$$
U \subseteq \mathbb{F}\left(a_{1}, \ldots, a_{d}\right)^{n+2} / \operatorname{Aut}\left(\mathbb{F}\left(a_{1}, \ldots, a_{d}\right)\right)
$$

and $\mathcal{F}(k)_{\underline{a}}$ (respectively $\mathcal{F}(h)_{\underline{a}}$ ) is the image of the incidence correspondence $I_{k}$ (resp. $\left.I_{h}\right)$ modulo $\operatorname{Aut}\left(\mathbb{F}\left(a_{1}, \ldots, a_{d}\right)\right)$.

But we can choose an affine covering so that in local coordinates, both $I_{k}$ and $I_{h}$, are two affine and transverse subspaces (modulo actions of $\mathbb{C}^{*}$ ).

In particular, since $\operatorname{dim} \mathcal{F}(k)_{\underline{a}}+\operatorname{dim} \mathcal{F}(h)_{\underline{a}} \geq \mathcal{F}(S)_{\underline{a}}$ by Proposition 4.9] we may conclude that

$$
\begin{aligned}
\operatorname{dim}\left(\mathcal{F}(k)_{\underline{a}} \cap \mathcal{F}(h)_{\underline{a}}\right) & =\operatorname{dim} \mathcal{F}(k)_{\underline{a}}+\operatorname{dim} \mathcal{F}(h)_{\underline{a}}-\mathcal{F}(S)_{\underline{a}} \\
& =2 n-3-\left(\operatorname{dim} \operatorname{Aut}(\underline{a})-\frac{n^{2}}{4}\right)
\end{aligned}
$$

But it is shown in Equation (1) of Theorem 3.1 that $\operatorname{dim} \operatorname{Aut}(\underline{a})-\frac{n^{2}}{4} \geq 0$, hence we obtain our inequality

\section{General binary curves and scrolls}

A binary curve is a stable curve $C=\Gamma_{1} \cup \Gamma_{2}$ where $\Gamma_{i} \cong \mathbb{P}^{1}$ for $i=1,2$, and the two curves intersect transversally in $n+2$ distinct points. Note that the arithmetic genus of $C$ is $p_{a}(C)=1-\chi\left(\mathcal{O}_{C}\right)=n+1$.

As usual by $K_{C}$ we denote a canonical divisor.

We denote by $\operatorname{Bin}_{p_{a}(C)} \subset \overline{\mathcal{M}}_{p_{a}(C)}$ the locus of binary curves in the moduli space fo genus $p_{a}(C)$ stable curves. By a general binary curve we mean a curve $C$ such that $[C]$ is general in $\operatorname{Bin}_{p_{a}(C)}$. 
As in the smooth case a first way to stratify binary curves is via the notion of gonality:

Definition 5.1 The gonality of $C$, gon $(C)$, is the smallest number $k$ such that there is a finite morphism $C \rightarrow \mathbb{P}^{1}$ of degree $k$. A curve $C$ is said to be hyperelliptic if $\operatorname{gon}(C)=2$.

First note that a general binary curve of genus $p_{a}(C) \geq 3$ is not hyperelliptic. Indeed if a binary curve is hyperelliptic then there is an isomorphism between the two components fixing the $p_{a}(C)+1$ points, which exists generically if and only if $p_{a}(C) \leq 2$.

Conversely, if $C$ is not hyperelliptic then $K_{C}$ is very ample on $C$ by 3 , Theorem 3.6] and $\varphi_{\left|K_{C}\right|}$ embeds $C$ in $\mathbb{P}^{n}$ as the union of two rational normal curves intersecting in $n+2$ distinct points in linear general position. Moreover by [9] $C$ satisfies Noether's Theorem, which means that the restriction maps

$$
H^{0}\left(\mathbb{P}^{n}, \mathcal{O}_{\mathbb{P}^{n}}(k)\right) \rightarrow H^{0}\left(C, \omega_{C}^{\otimes k}\right)
$$

are surjective for every $k \geq 0$.

Therefore we can identify $C=\Gamma_{1} \cup \Gamma_{2}$ and the points $\Gamma_{1} \cap \Gamma_{2}=\left\{P_{0}, \cdots, P_{n+1}\right\}$ with their canonical embeddings in $\mathbb{P}^{n}$. This way, $C$ is a general binary curve if and only if $\Gamma_{1}$ and $\Gamma_{2}$ are general rational normal curves passing through $\left\{P_{0}, \cdots, P_{n+1}\right\}$.

We have the following useful remark.

Remark 5.2 Let $C=\Gamma_{1} \cup \Gamma_{2}$ be a general binary curve canonically embedded in $\mathbb{P}^{n}$ and let $P_{0} \in \Gamma_{1} \cap \Gamma_{2}$.

Consider the projection from $P_{0}$ onto a general hyperplane and write it as

$$
\pi_{P_{0}}: \mathbb{P}^{n} \rightarrow \mathbb{P}^{n-1} \text {. }
$$

Then $C^{\prime}=\overline{\pi_{P_{0}}(C)}$ is a general binary curve of arithmetic genus $p_{a}\left(C^{\prime}\right)=$ $p_{a}(C)-1$ canonically embedded in $\mathbb{P}^{n-1}$.

Now let us point out that it is easy to prove that the usual bound on the gonality of smooth curves holds for binary curves as well.

Lemma 5.3 Let $C=\Gamma_{1} \cup \Gamma_{2}$ be a binary curve of arithmetic genus $p_{a} \geq 3$. Then

$$
\operatorname{gon}(C) \leq\left\lfloor\frac{p_{a}(C)+3}{2}\right\rfloor
$$

Proof. Let $\Gamma_{1} \cap \Gamma_{2}=\left\{P_{0}, \cdots P_{n+1}\right\}$, where $n=p_{a}(C)-1$. The Lemma follows if we show that there exists a morphism $\psi: \Gamma_{1} \rightarrow \Gamma_{2}$ of degree $\left\lfloor\frac{n}{2}+1\right\rfloor$ which is the identity restricted to $\Gamma_{1} \cap \Gamma_{2}$. Indeed, the morphism $\varphi: C \rightarrow \mathbb{P}^{1}$ defined as $\psi$ on $\Gamma_{1}$ and the identity on $\Gamma_{2}$ is well defined and has the required degree.

To show the existence of such $\psi$ let us consider the normalisation $\pi: \hat{C} \cong$ $\Gamma_{1} \sqcup \Gamma_{2} \rightarrow C$ and let $\left\{R_{i}, S_{i}\right\}=\pi^{*}\left(P_{i}\right)$ where $R_{i} \in \Gamma_{1}$ and $S_{i} \in \Gamma_{2}$. 
Assume at first that $n$ is even.

Taking coordinates on $\Gamma_{2} \cong \mathbb{P}^{1}$ such a map should be given by a pair of polynomials of degree $\frac{n}{2}+1, \psi=\left(q_{1}, q_{2}\right)$ and the conditions of being the identity on the intersection correspond to ask $\psi\left(R_{i}\right)=S_{i}$ for $i=1, \ldots, n+2$. Now, the vector space of couples $\left(q_{1}, q_{2}\right)$ with the required degree has dimension $2 \cdot\left(\frac{n}{2}+2\right)$, while every pair $\left(R_{i}, S_{i}\right)$ imposes one single condition on such vector space. In particular there is a 2-dimensional vector space of $\left(q_{1}, q_{2}\right)$ with the required properties, i.e., there exists a one dimensional family of such maps $\psi$.

Similarly, if $n$ is odd we may find a map $\psi: \Gamma_{1} \rightarrow \Gamma_{2}$ of degree $\left\lfloor\frac{n}{2}+1\right\rfloor$ which is the identity restricted to $\Gamma_{1} \cap \Gamma_{2}$.

Remark 5.4 Let $C=\Gamma_{1} \cup \Gamma_{2}$ be a binary curve of arithmetic genus $p_{a} \geq 3$. We can define its Clifford index (as in the smooth case) as follows

$$
\operatorname{Cliff}(C)=\min _{L \in \operatorname{Pic}(C)}\left\{\operatorname{deg}(L)-2 h^{0}(C, L)+2: h^{0}(C, L) \geq 2, h^{1}(C, L) \geq 2\right\}
$$

(see 8 for a generalisation to singular curves).

In the smooth case it is a classical result that the gonality is maximal if and only if the Clifford index is maximal, i.e.

$$
\operatorname{gon}(C)=\left\lfloor\frac{p_{a}(C)+3}{2}\right\rfloor \Longleftrightarrow \operatorname{Cliff}(C)=\left\lfloor\frac{p_{a}(C)-1}{2}\right\rfloor
$$

We point out that the same holds for binary curves.

Indeed, it is straightforward to see that $\operatorname{Cliff}(C) \leq \operatorname{gon}(C)-2$. Conversely we may apply verbatim [5. Theorem 2.3] and see that if the Clifford index is not maximal then there is a one-dimensional family of maps $C \rightarrow \mathbb{P}^{1}$ of degree $\leq\left\lfloor\frac{p_{a}(C)+3}{2}\right\rfloor$; the same arguments used in Lemma 5.3 show that there is at least one map $C \rightarrow \mathbb{P}^{1}$ of degree $\leq\left\lfloor\frac{p_{a}(C)+1}{2}\right\rfloor$.

To conclude we show that for a general binary curves there is no scroll of small dimension containing it.

Theorem 5.5 Let $C=\Gamma_{1} \cup \Gamma_{2}$ be a general binary curve in $\mathbb{P}^{n}$.

Then there is no rational normal scroll of minimal degree and of dimension $\leq \frac{n}{2}$ containing $C$.

Proof. Let $C=\Gamma_{1} \cup \Gamma_{2}$ and let $\Gamma_{1} \cap \Gamma_{2}=\left\{P_{0}, \cdots, P_{n+1}\right\}$. We argue by induction on $n$. If $n=3$ the result is trivial.

Assume that $\mathbb{F}$ is a scroll of dimension $d$ containing $C$. For $n \geq 4$ we are going to consider the projection from a point $P_{0} \in \Gamma_{1} \cap \Gamma_{2}$ onto a general hyperplane

$$
\pi_{P_{0}}: \mathbb{P}^{n} \rightarrow \mathbb{P}^{n-1} \text {. }
$$

We have that $C^{\prime}=\pi_{P_{0}}(C)$ is again a binary curves (by Remark [5.2) contained in $\mathbb{F}^{\prime}=\pi_{P_{0}}(\mathbb{F})$ which is a scroll of dimension $\leq d$.

In particular if $d \leq \frac{n-1}{2}$ the theorem follows by induction. 
Therefore we are left with the case where $C \subset \mathbb{F}$ a scroll of dimension $d=\frac{n}{2}$, where $n$ is an even integer.

The theorem follows if we show that under these hypotheses either there exists a scroll $\mathbb{G} \subset \mathbb{F}$ of lower dimension containing $C$, or $\mathbb{F}$ is singular at one of the points in $\Gamma_{1} \cap \Gamma_{2}$, say $P_{0}$. Indeed in the first case $\operatorname{dim}(\mathbb{G})<\frac{n-1}{2}$ whereas in the second case taking the projection from the singular point $P_{0}$ we obtain a scroll $\mathbb{F}^{\prime}=\pi_{P_{0}}(\mathbb{F}) \subset \mathbb{P}^{n-1}$ of dimension $\leq \frac{n-1}{2}$ containing $C^{\prime}=\pi_{P_{0}}(C)$.

So let $C=\Gamma_{1} \cup \Gamma_{2}$ be a general binary curve and assume that there exists a scroll $\mathbb{F}\left(a_{1}, \ldots, a_{d}\right) \rightarrow \mathbb{F} \subset \mathbb{P}^{n}$ of degree $\frac{n}{2}+1$ and dimension $\frac{n}{2}$ containing $C$. Let $L, M$ be the pullback of $\mathcal{O}_{\mathbb{P}^{1}}(1)$, respectively $\mathcal{O}_{\mathbb{P}^{n}}(1)$. It is clear that $|L|$ induces two maps

$$
\Gamma_{1} \stackrel{h: 1}{\longrightarrow} \mathbb{P}^{1} \quad \Gamma_{2} \stackrel{k: 1}{\longrightarrow} \mathbb{P}^{1} .
$$

Note that $h \leq \frac{n}{2}$ and $k \leq \frac{n}{2}$ since $\operatorname{dim}(\mathbb{F})=\frac{n}{2}$.

If $h+k<\frac{n+2}{2}$ then by [13, Chapter 2] and Riemann-Roch for $C$ we have

$$
h^{0}\left(\mathbb{F}, \mathcal{O}_{\mathbb{F}}(L+M)\right)=\frac{n+2}{2}+n>h^{0}\left(C, \mathcal{O}_{C}(L+M)\right)=h+k+n
$$

i.e., there exists $\mathbb{G} \in|L+M|$ containing $C$. Such a $\mathbb{G}$ is linearly equivalent to a rational normal scroll and it is irreducible since otherwise every proper component should be degenerate because of the minimality of $\operatorname{deg}(\mathbb{G})$, contradicting $C$ non-degenerate. Therefore if $h+k<\frac{n+2}{2}$ there exists a scroll $\mathbb{G}$ of dimension $\leq \frac{n}{2}-1$ containing $C$.

If $h+k \geq \frac{n+2}{2}$ and $h \neq k$ we take the following families

- $\mathcal{B}(S):=$ the family of binary curves passing through a set $S$ consisting of $n+2$ fixed points in linear general position $\left\{P_{0}, \cdots, P_{n+1}\right\}$;

- $\mathcal{F}_{h, k}=\mathcal{F}(S, k)_{\underline{a}} \cap \mathcal{F}(S, h)_{\underline{a}}:=$ the family of scrolls of dimension $\frac{n}{2}$ in $\mathbb{P}^{n}$ containing a binary curve passing trough $\left\{P_{0}, \cdots, P_{n+1}\right\}$ with induced maps onto $\mathbb{P}^{1}$ of degree $h$, resp. $k$.

Consider now the incidence correspondence

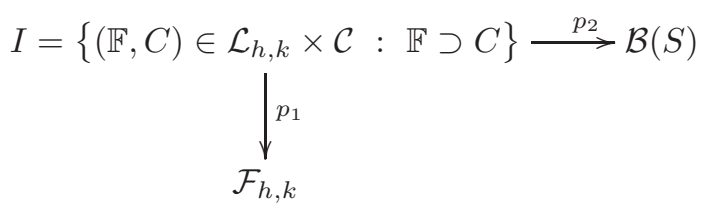

Recall that $\operatorname{dim} \mathcal{B}(S)=2 n-2$ by Theorem 3.1 and that $\operatorname{dim} \mathcal{F}_{h, k} \leq 2 n-3$ by Proposition 4.10 
If for a general binary curves there exists a $d$-dimensional scroll containing it then $p_{2}$ must be dominant. In particular $\operatorname{dim} I \geq \operatorname{dim} \mathcal{B}(S)=2 n-2$ and moreover the generic fibre of the map $p_{1}$ must have positive dimension.

Therefore, fixing $P_{0}, \cdots, P_{n+1}$ and $\mathbb{F}$, our dimension count shows in particular that there exists a positive dimension family of rational normal curves in $\mathbb{F}$ containing $\Gamma_{1} \cap \Gamma_{2}=\left\{P_{0}, \cdots, P_{n+1}\right\}$. Therefore by Proposition 4.9 and Proposition 4.6 we may conclude that one of the points in $\Gamma_{1} \cap \Gamma_{2}$ is contained in $\operatorname{Sing}(\mathbb{F})$ and we can conclude.

If $h+k \geq \frac{n+2}{2}$ and $h=k$ then by Proposition 4.8 there exists again a positive dimensional family of rational normal curves passing through the $n+2$ points, hence by Prop. 4.6 and Prop. $4.8 \mathbb{F}$ is singular at one of the points in $\Gamma_{1} \cap \Gamma_{2}$, and we can conclude.

\section{References}

[1] M.Aprodu and J. Nagel, Koszul cohomology and algebraic geometry, University Lecture Series, 52, American Mathematical Society, Providence, RI, (2010),

[2] F. Bogomolov and V. S. Kulikov, On the irreducibility of Hilbert scheme of surfaces of minimal degree, Cent. Eur. J. Math. 11 (2013), no. 2, 254-263.

[3] F. Catanese, M. Franciosi, K. Hulek, and M. Reid, Embeddings of curves and surfaces, Nagoya Math. J. 154 (1999), 185-220.

[4] E. Colombo and P. Frediani, On the koszul cohomology of canonical and prym-canonical binary curves, Bull. London Math. Soc. 45 (2013), 10311040.

[5] M.Coppens and G.Martens Secant spaces and Clifford's theorem, Compositio. Math. 78 (1991), no.2, 193-212.

[6] D. Eisenbud, The geometry of syzygies, Graduate Texts in Mathematics, vol. 229, Springer-Verlag, New York, 2005.

[7] D. Eisenbud and J. Harris, On varieties of minimal degree (a centennial account), Algebraic geometry, Bowdoin, 1985 (Brunswick, Maine, 1985), Proc. Sympos. Pure Math., vol. 46, Amer. Math. Soc., 1987, pp. 3-13.

[8] M. Franciosi and E. Tenni, On Clifford's theorem for singular curves, Proc. Lond. Math. Soc. 108 (2014), no. 3, 225-252.

[9] M. Franciosi and E. Tenni, The canonical ring of a 3-connected curve, Atti Accad. Naz. Lincei Rend. Lincei Mat. Appl., 25, (2014), no. 1, 37-51,

[10] J. Harris, Algebraic geometry, A first course, Graduate Texts in Mathematics 133, 1992. 
[11] R. Hartshorne, Algebraic geometry, Springer-Verlag, New York, 1977, Graduate Texts in Mathematics 52, 1977.

[12] J. O. Kleppe and R. M. Miró-Roig, Dimension of families of determinantal schemes, Trans. Amer. Math. Soc. 357 (2005), no. 7, 2871-2907.

[13] M. Reid, Chapters on algebraic surfaces, Complex algebraic geometry (Park City, 1993) (J. Kollár, ed.), IAS/Park City Math. Ser., vol. 3, Amer. Math. Soc., 1997, pp. 3-159.

\author{
Marco Franciosi \\ Dipartimento di Matematica, Università di Pisa \\ Via Buonarroti 1, I-56127 Pisa (Italy) \\ marco.franciosi@unipi.it
}

